\title{
KDIGO Guidelines for Care of the Kidney Transplant Recipient
}

\author{
A. Khwaja \\ Sheffield Kidney Institute, Northern General Hospital, Sheffield, UK
}

Clinical practice guidelines from Kidney Disease Improving Global Outcomes (KDIGO) relating to the care of the kidney transplant patient were published in 2009 [1]. These guidelines encompass all aspects of transplant care and focus on five key areas: immunosuppression, graft monitoring and infections, cardiovascular disease, malignancies, and other complications including bone disease, anaemia, fertility and sexual function.

KDIGO has utilised their standard grading system for clinical practice recommendations with the strength of recommendation being graded as level 1 or level 2 with level 1 being a 'we recommend...' statement implying most patients should receive this action whilst level 2 is a 'suggestion' recognising that management decisions may vary in different clinical contexts. Each recommendation was further graded from $A$ to $D$ by the quality of evidence underpinning them with grade A referring to a high quality of evidence whilst grade D recognised a 'very low' evidence base.

Selected key aspects of the guideline are summarised in table 1 . In addition, the guidelines recognise the financial barriers to transplantation and globally suggest clinical strategies to reduce drug costs in settings where drug costs block access to transplantation. These include limiting the use of induction agents and mycophenolate, minimising the dose of calcineurin inhibitors by routine use of ketoconazole or nondihydropyridine calcium channel blockers, using adequately tested bioequivalent generics and long-term use of prednisolone. These comprehensive guidelines also identify key areas of future research highlighting the need for randomised controlled trials to determine the value of steroid avoidance, the utility of protocol biopsies, the optimal treatment of antibody-mediated rejection and interventions to improve medication adherence. In striking contrast to the KDIGO Chronic Kidney Disease-Mineral Bone Disorder guideline [2], the transplant guidelines are based on a much more robust evidence base and will be an invaluable guide to the practicing transplant clinician.

\footnotetext{
References 1 Kidney Disease: Improving Global Outcomes (KDIGO) Transplant Work Group: KDIGO clinical practice guideline for the care of kidney transplant recipients. Am J Transplant 2009;suppl 3:S1-S155.

2 Kidney Disease: Improving Global Outcomes (KDIGO) CKD-MBD Work Group: KDIGO clinical practice guideline for the diagnosis, evaluation, prevention, and treatment of Chronic Kidney Disease-Mineral and Bone Disorder (CKD-MBD). Kidney Int Suppl 2009;113:S1-S130.
} 
Table 1. Care of kidney transplant recipients

\begin{tabular}{|c|c|}
\hline $\begin{array}{l}\text { Immunosuppression } \\
\text { Induction }\end{array}$ & $\begin{array}{l}\text { - IL-2 receptor antagonist first-line agent } \\
\text { - lymphocyte-depleting agent for high immunological risk }\end{array}$ \\
\hline Maintenance & $\begin{array}{l}\text { - CNI and antiproliferative agent } \\
\text { - tacrolimus preferable to cyclosporin } \\
\text { - } \text { mycophenolate preferable to azathioprine } \\
\text { - } \text { stop steroids within a week if low immunological risk } \\
\text { - CNI to be continued in the long term } \\
\text { - } \text { only use mTORi if stable graft function and wound healed } \\
\text { - } \text { monitor CNI, mycophenolate and mTORi levels }\end{array}$ \\
\hline Acute rejection & $\begin{array}{l}\text { - } \text { acute cellular rejection treat with steroids even if borderline } \\
\text { - OKT3/lymphocyte depleting antibody for recurrent or steroid resistance } \\
\text { - } \text { antibody-mediated rejection one or more of: plasma exchange, immunoglobulin, } \\
\text { anti-CD20 antibody, lymphocyte-depleting antibody, } \pm \text { steroids } \\
\text { - } \text { maintenance mycophenolate }\end{array}$ \\
\hline Chronic allograft injury & $\begin{array}{l}\text { - } \text { biopsy if cause unclear } \\
\text { - } \text { minimise/withdraw CNI if CNI toxicity } \\
\text { - } \text { eGFR }>40 \mathrm{ml} / \mathrm{min} / 1.73 \mathrm{~m}^{2} \text { and proteinuria }<500 \mathrm{mg} / \text { day replace CNI with } \mathrm{mTORi}\end{array}$ \\
\hline \multicolumn{2}{|l|}{ Monitoring } \\
\hline Biopsy indications & $\begin{array}{l}\text { - unexplained graft dysfunction } \\
\text { - delayed graft function } \\
\text { - new-onset proteinuria or unexplained proteinuria }>3 \mathrm{~g} / 24 \mathrm{~h}\end{array}$ \\
\hline Recurrent disease & - monitor for recurrence of FSGS, MPGN, IgA, HUS, vasculitis, oxaluria \\
\hline \multicolumn{2}{|l|}{ Viruses } \\
\hline BK polyoma virus & $\begin{array}{l}\text { - interval screening with plasma PCR for } 12 \text { months/if unexplained graft dysfunction/after treatment } \\
\text { of acute rejection } \\
\text { - } \text { reduce immunosuppression if PCR }>10 \text { copies/ml }\end{array}$ \\
\hline EBV & $\begin{array}{l}\text { - monitor PCR in donor-seropositive recipient-seronegative transplants } \\
\text { - } \text { reduce/stop immunosuppression if EBV disease or post-transplant lymphoproliferative disease }\end{array}$ \\
\hline $\mathrm{HCV}$ and $\mathrm{HBV}$ & $\begin{array}{l}\text { - conventional immunosuppression for all HBV/HCV recipients } \\
\text { - interferon only for life-threatening liver disease } \\
\text { - interval screening for cirrhosis, hepatocellular carcinoma and proteinuria } \\
\text { - } \text { prophylaxis for HBsAg-positive recipients: tenofovir/entecavir/lamivudine }\end{array}$ \\
\hline $\begin{array}{l}\text { Metabolic/cardiovascular } \\
\text { disease }\end{array}$ & $\begin{array}{l}\text { - } \text { frequent interval screening for new-onset diabetes for } 1 \text { year, then annually } \\
\text { - } \text { target blood pressure }<130 / 80 \mathrm{~mm} \mathrm{Hg} \\
\text { - } \text { ACEi/ARB if proteinuria }>1 \mathrm{~g} / 24 \mathrm{~h} \\
\text { - } \text { treat dyslipidaemia based on KDOQI guidelines }\end{array}$ \\
\hline Malignancy & $\begin{array}{l}\text { - consider reducing immunosuppression } \\
\text { - use mTORi for Kaposi's sarcoma }\end{array}$ \\
\hline \multicolumn{2}{|c|}{$\begin{array}{l}\text { IL-2 = Interleukin-2; CNI = calcineurin inhibitor; } \mathrm{mTORi}=\mathrm{mTOR} \text { inhibitor; eGFR = estimated GFR; FSGS = focal segmental glo- } \\
\text { merulosclerosis; } \mathrm{MPGN}=\text { mebranoproliferative glomerulonephritis, } \mathrm{HUS}=\text { haemolytic uraemic syndrome; } \mathrm{PCR}=\text { polymerase chain } \\
\text { reaction; EBV = Epstein-Barr virus; HCV = hepatitis C virus; HBV = hepatitis B virus; ACEi = angiotensin converting enzyme; } \\
\text { ARB = angiotensin receptor blocker. }\end{array}$} \\
\hline
\end{tabular}

